\title{
Real-time tissue elastography for testicular lesion assessment
}

\author{
Alfredo Goddi • Andrea Sacchi • Giovanni Magistretti • \\ Joan Almolla • Maurizio Salvadore
}

Received: 27 April 2011 /Revised: 31 August 2011 /Accepted: 3 September 2011 /Published online: 26 October 2011

(C) The Author(s) 2011. This article is published with open access at Springerlink.com

\begin{abstract}
Objectives To assess the ability of Real-time Elastography (RTE) to differentiate malignant from benign testicular lesions.

Methods In 88 testicles ultrasound identified 144 lesions, which were examined by RTE. Elasticity images of the lesions were assigned the colour-coded score of Itoh (Radiology 2006), according to the distribution of strain induced by light compression. RTE findings were analysed considering shape (nodular/pseudo-nodular), size $(<5 \mathrm{~mm}$, 6-10 mm, >11 mm) and score (SC1-5) of the lesions.

Results $93.7 \%$ of all benign lesions showed a complete elastic pattern ( $\mathrm{SC} 1$ ). $92.9 \%$ of benign nodules $<5 \mathrm{~mm}$ and $100 \%$ of the pseudonodules showed a nearly complete elastic pattern (mainly $\mathrm{SC} 1$ ). $87.5 \%$ of malignant nodules showed a stiff pattern (SC4-5). RTE gave $87.5 \%$ sensitivity, 98.2\% specificity, $93.3 \%$ positive predictive value, $96.4 \%$ negative predictive value and $95.8 \%$ accuracy in differentiating malignant from benign lesions.
\end{abstract}

A. Goddi $(\bowtie) \cdot$ A. Sacchi $\cdot$ G. Magistretti

SME-Diagnostica per Immagini Medical Center,

Via L. Pirandello, 31,

21100 Varese, VA, Italy

e-mail: goddi.alfredo@libero.it

\section{J. Almolla}

Institute of Radiology, San Matteo Hospital IRCCS,

University of Pavia,

Piazzale Golgi,

27100 Pavia, Italy

M. Salvadore

Department of Pathology,

University Hospital and Fondazione Macchi,

Viale Borri, 57,

21100 Varese, Italy
Conclusions RTE is a useful technique in assessing small testicular nodules and pseudo-nodules. This is relevant in clinical practice allowing expectant management in RTE selected cases. The role of RTE seems less relevant for larger lesions because most of them are malignant at clinical and ultrasound assessment, limiting RTE to simply confirmation role.

Key Points

- An emerging role for Elastography in allowing surveillance for small testicular lesions

- Elastography can better differentiate benign from malignant testicular lesions

- Follow up can be reduced for elastic testicular lesions at Elastography.

Keywords Elastography · Sonoelastography · Ultrasound · Scrotum $\cdot$ Testis

\section{Introduction}

Until the advent of B-mode ultrasound, diagnosis of scrotal lesions relied on palpation and their characterisation was based on the detection of changes in tissue consistency. Considering the limitations of clinical assessment, ultrasound has so far been considered the gold standard for evaluating scrotal abnormalities [1]. Ultrasound is extremely sensitive in the detection of testicular masses, but does not provide a histological diagnosis [2].

Sonoelastography has been recently introduced for making noninvasive measurements of the mechanical properties of tissue and for imaging the elasticity of biological tissue [3, 4]. It represents a "new way" of palpation, where a portion of tissue is compressed and the degree to which it displaces is assessed. The most common way to displace the tissue is by 
manually applying a slight longitudinal compression with a conventional probe (so called "strain imaging"): it is expected that different tissues create different responses according to their specific elastic modulus [5].

Real-time Elastography (RTE) was the first commercial ultrasound technique available for wide clinical applications. RTE evaluates the relative elasticity of different tissues in a selected region of interest by using a fast crosscorrelation technique and a combined autocorrelation method. It creates an elastogram that is superimposed to the B-mode ultrasound image of the tissue and updated in real-time $[5,6]$. By convention, the elastograms display a colour-coded map of the relative elasticity (stiffer areas are depicted as blue and softer areas are red; green indicates an intermediate level of elasticity).

Considering that most solid tumours differ in their consistency from the surrounding or deriving tissue, RTE may offer a useful tool for characterising different lesions. Elastosonography has been proven to be a highly specific imaging technique for the clinical assessment of breast, prostate, thyroid and lymph node lesions [6-13]. Few reports concerning the efficacy of RTE for scrotal mass assessment are available [14-16].

The objective of this preliminary study is to assess RTE ability to discriminate malignant from benign testicular lesions.

\section{Materials and methods}

Between April 2007 and December 2010, 1617 patients (mean age: 34 years - range: 2 months -89 years) were referred to our Medical Centre Ultrasound Unit for scrotal abnormalities. A total 3173 testicles were examined by ultrasound. In $324 / 3173$ of the testicles a structural lesion was identified. Ultrasound alone was able to define the "non-tumoral" aspect of 236/324 of these testicles (Table 1) and to avoid further examinations.

The others 88/324 testicles, in which we identified 144 mainly solid lesions, were examined by colour Doppler (CD) and RTE in an attempt to differentiate between malignant and benign nature.

Table 1 "Non-tumoural" findings in 3173 testicles

\begin{tabular}{ll}
\hline Small isolated calcification & 43 \\
Scattered small calcifications (less than \# 5) & 18 \\
Microlithiasis & 42 \\
Cyst $<5 \mathrm{~mm}$ & 78 \\
Cyst from 6 to $10 \mathrm{~mm}$ & 7 \\
Cyst $>11 \mathrm{~mm}$ & 4 \\
Intratesticular spermatocele & 44 \\
Total & 236 \\
\hline
\end{tabular}

The study focuses on the focal testicular lesions; thus the more common acute inflammatory disease of the testis are not considered in this study. The local institutional review board approved the study.

The patients were informed about the advantages and limitations offered by RTE and informed consent was obtained before undergoing the ultrasound examination. The ultrasound and RTEs were performed by three consultant radiologists with more than 20 years' experience in scrotal ultrasound. RTE assessment lasted between 1 and $7 \mathrm{~min}$, and the average time spent on assessment was $3 \mathrm{~min}$.

Patients with suspect testicular malignant lesions were referred for surgery and histological evaluation. In accordance with the consultant urologist, periodic follow up - every 3 or 6 months - was planned for patients with lesions not suspicious for malignancy.

Two different ultrasound systems were used for this study: the Hi Vision EUB 8500 and the Preirus (Hitachi Medical, Tokyo, Japan), both equipped with a $5-13-\mathrm{MHz}$ and $6-14-\mathrm{MHz}$ linear probe.

After basic examination in B-Mode, the mode was switched to Elastography. A medium or wide size elastogram box (RTE-box) was usually used to increase the number of samples for the average strain calculation; usually it does not include the scrotal wall to avoid misinterpretation due to their relative stiff aspect. RTE was performed exerting manually a very slight pressure on the testicle using the linear probe. The pressure intensity was adapted to the on-screen quality indicator scale providing the user with real-time feedback on the appropriate stress. It ranges from 1 to 5 levels on the 'Hitachi' units; as a reference we used a value of 3 (Fig. 1). The combination of the different parameters set for the scrotal application allowed a RTE frame rate of $13 \mathrm{~Hz}$.

RTE images of the lesions were assigned an elastographic score (SC1 to 5) according to the distribution and degree of strain suggested by Itoh et al. for breast disease [6]. If a lesion was scored between 1 and 3 , it would be benign; if a lesion was scored 4 or 5 , it would be considered malignant. A score of 1 indicated even strain for the entire lesion. A score of 2 indicated strain in most of the solid lesion with some areas of no strain. A score of 3 indicated strain at the periphery of the solid lesion, with no strain at the centre of the lesion. A score of 4 indicated no strain in the entire lesion. A score of 5 indicated no strain in the entire solid lesion and in the surrounding area.

Diagnostic sensitivity, specificity, positive predictive value (PPV), negative predictive value (NPV) and accuracy in differentiating malignant from benign lesions were assessed comparing the findings with histological reports or with the results of a close follow-up of almost 1 year.

In the case of malignancy, true-positive (TP) cases were lesions with an elasticity score of 4 or 5 and histological 

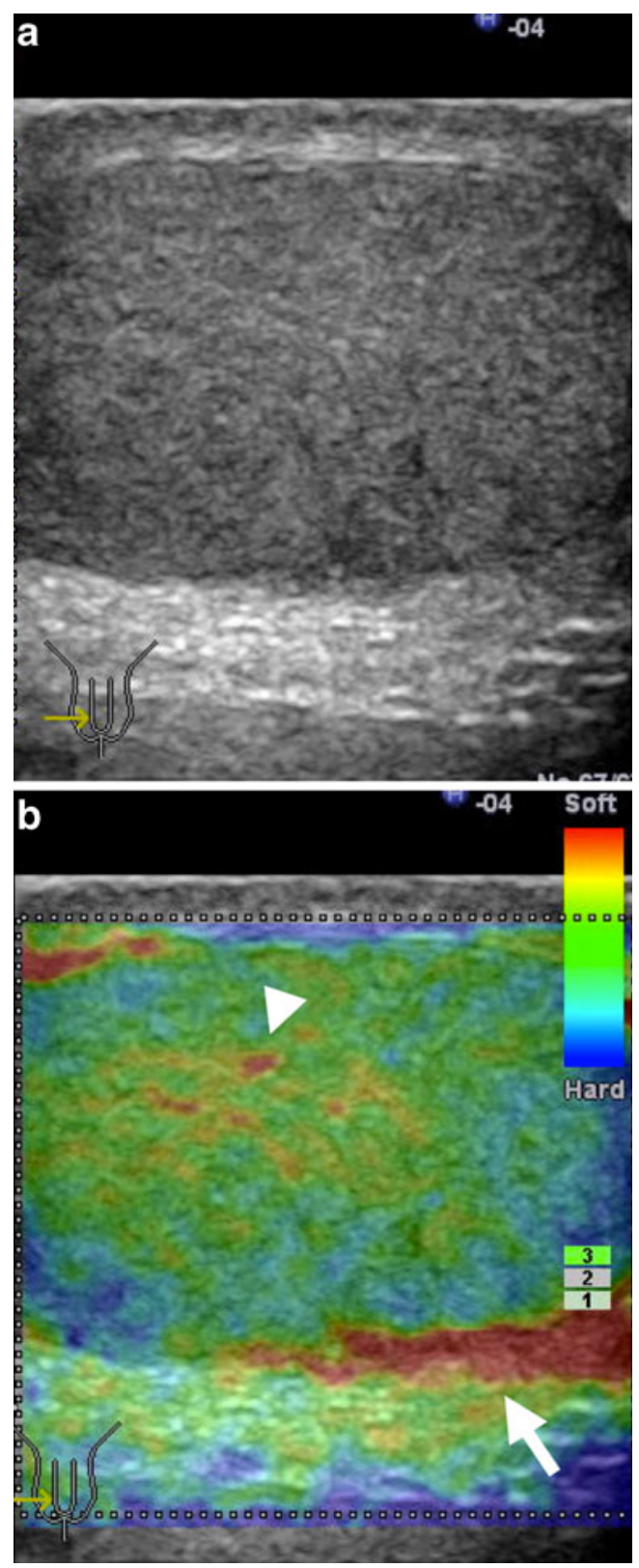

Fig. 1 a Grey-scale ultrasound longitudinal image of the testis: normal homogeneous fine granular echo pattern. b Elastography: a wide RTE box was selected to evaluate testicular elasticity. The typical distribution of elasticity in a normal testis shows mainly green (intermediate), some linear red (soft) (arrowheads) and peripheral light blue (stiff); outside the testicle RTE shows red (soft) related to the fluid in the intravaginal space (arrow). On the right side the quality scale indicating levels 3

confirmation; true-negative (TN) cases were those with a score of 1,2 or 3 found to be benign at histology or at prolonged follow-up. False-positive (FP) lesions were those with a score of 4 or 5 that were found to be benign at histology or at prolonged follow-up. False-negative (FN) cases were defined as lesions with a score of 1,2 or 3 and a diagnosis of malignancy at histology.
The data analysis of the lesions taking into account even their shape - typically nodular or pseudo-nodular - and especially their size $(<5 \mathrm{~mm}, 6-10 \mathrm{~mm},>11 \mathrm{~mm})$.

\section{Results}

The RTE findings, analysed considering shape, size, elastographic criteria (SC1-5) and the histological diagnosis or the benign appearance in the follow up examinations of the 144 lesions identified, are shown in Table 2.

According to the elastographic criteria, RTE (Table 3) showed 106 lesions with score 1 (Fig. 2a), 105 of which were confirmed as benign after the close follow-up and one which was diagnosed to be a Leydig tumour at histology. Eight lesions were classified as score 2: 5 of them were benign, 1 was a Leydig tumour (Fig. 3), 1 was a small seminoma and 1 was a teratocarcinoma. There were no testicular lesions with score 3 identified in our group of patients. $27 / 28$ of the lesions with score 4 were found at histology to be malignant and 1 that behaved as a benign nodule $(5 \mathrm{~mm})$ after 30 months of close follow-up. Finally, only one of the two score 5 lesions was malignant; the other after close monitoring was shown to be a focal posttraumatic haematoma (Fig. 4). $87.5 \%$ of the malignant lesions showed stiff patterns (SC4-5).

The results of the elastograms in relation to the lesion shape are shown in Table 4.

The results of the elastograms regarding the nodule size are shown in Table 5. In the first sub-group (nodules $<5 \mathrm{~mm}$ ), RTE defined as elastic (64 SC1 and $2 \mathrm{SC} 2$ ), therefore benign, 66/71 (92.9\%) nodules. The follow-up at 24 months has not shown any variation of the elastic signal or a significant increase in size, confirming therefore the initial diagnosis. Five nodules were completely "inelastic" (5 SC4) and therefore were considered malignant. Four of them were found in cases of multifocal seminomas (histological diagnosis). The latter was a single nodule (Fig. 2b); considering the small size, in accordance with the consultant urologist, a close follow-up was planned (reassessment every 3 months). After 30 months the lesion had remained unchanged, therefore the nodule was reclassified as "probably benign" and its inelastic aspect was considered a falsepositive of the RTE method.

In the second sub-group (nodules $6-10 \mathrm{~mm}$ ), 19 of the 21 nodules were correctly characterised: 4 of the 19 nodules (2 SC1 and 2 SC2) didn't show any variation at the 24 months follow-up and 15 nodules (all SC4) were malignant at the histological diagnosis. In $2 / 21$ the diagnosis was missed. The first of them was an isoechoic nodule $(6 \mathrm{~mm})$ with a hypoechoic thin rim, characterised by a nearly complete elastic pattern, and therefore possibly a benign lesion at the RTE evaluation. Considering the B- 
Table 2 RTE findings compared to the histological diagnosis and the benign appearance in the follow up examinations in 144 nodules/pseudonodules

\begin{tabular}{|c|c|c|c|c|c|c|c|c|}
\hline & \multirow[t]{2}{*}{$\#$ of lesions } & \multicolumn{5}{|c|}{ Real-time elastography } & \multicolumn{2}{|c|}{ Diagnosis } \\
\hline & & Score 1 & Score 2 & Score 3 & Score 4 & Score 5 & Benign & Malignant \\
\hline Nodules $<5 \mathrm{~mm}$ & 71 & 64 & 2 & - & 5 & - & 67 & 4 \\
\hline Nodules $6-10 \mathrm{~mm}$ & 21 & 3 & 2 & - & 15 & 1 & 4 & 17 \\
\hline Nodules $>11 \mathrm{~mm}$ & 11 & - & 2 & - & 8 & 1 & - & 11 \\
\hline Sub-Total & 103 & 67 & 6 & - & 28 & 2 & 71 & 32 \\
\hline $\begin{array}{l}\text { Pseudo-nodules }{ }^{\mathrm{a}} \\
<5 \mathrm{~mm}\end{array}$ & 4 & 4 & - & - & - & - & 4 & - \\
\hline $\begin{array}{l}\text { Pseudo-nodules }{ }^{\mathrm{a}} \\
6-10 \mathrm{~mm}\end{array}$ & 26 & 26 & - & - & - & - & 26 & - \\
\hline $\begin{array}{l}\text { Pseudo-nodules } \\
>11 \mathrm{~mm}\end{array}$ & 11 & 9 & 2 & - & - & - & 11 & - \\
\hline Sub-Total & 41 & 39 & 2 & & & & 41 & \\
\hline Total & 144 & 106 & 8 & 0 & 28 & 2 & 112 & 32 \\
\hline
\end{tabular}

${ }^{\mathrm{a}}$ linear - lobular - half-moon - undefined areas

mode ultrasound findings, the peripheral vascularisation at colour Doppler evaluation, and the development of gynaecomastia, associated with an abnormal endocrine profile, surgery was carried out. Conservative surgery was planned and performed on the basis of the RTE findings. The histological diagnosis was a Leydig tumour. This case was considered an RTE false-negative. The latter missed nodule was a subcapsular oval-shaped hypoechoic nodule in a patient with recent trauma. It showed score 5 at the RTE, indicating no strain in the entire lesion and in the surrounding area: this aspect is considered related to malignant tissue with perinodular infiltration in the breast applications, thus to avoid a misdiagnosis we chose to classify it as malignant. Considering the shape and the recent trauma, a haematoma could not be excluded with certainty; thus a strict follow-up was decided upon in accordance with the consultant urologist. The lesion did not grow and after 3 months the RTE hard signal in the surrounding area changed; after 6 months half of the nodule showed regular strain; after 9 months it was reduced in size and partially calcified: the appearance became elastic (score 1). This case must be considered an RTE falsepositive finding (Fig. 4).

Table 3 Elasticity scores in the 144 testicular nodules/pseudo-nodules. $28 / 32(87.5 \%)$ of the malignant lesions showed stiff pattern (SC4-5)

\begin{tabular}{llll}
\hline & Benign & Malignant & Total \\
\hline Score 1 & 105 & 1 & 106 \\
Score 2 & 5 & 3 & 8 \\
Score 3 & - & - & - \\
Score 4 & 1 & 27 & 28 \\
Score 5 & 1 & 1 & 2 \\
\hline
\end{tabular}

In the third sub-group (nodules $>11 \mathrm{~mm}), 9 / 11(81.8 \%)$ nodules were hypoechoic and showed "inelastic" pattern ( 8 $\mathrm{SC} 4$ and 1 SC5) at the RTE: they were found to be seminomas at surgery (Fig. 5). One of the two remaining nodules $(14 \mathrm{~mm})$, slightly hyperechoic compared with the surrounding tissue, presented a nearly complete stiff rim and a wide central elastic area (i.e. the peripheral part of the lesion was blue, the central part was green) and was classified as score 2. Even colour Doppler showed mainly peripheral vascularisation of the lesion. Despite these findings, considering the increased consistency at clinical palpation and the lesion size, surgery was carried out. Histological diagnosis was Leydig tumour, resulting in a RTE false-negative finding (Fig. 3). The other nodule (30× $25 \times 17 \mathrm{~mm}$ ), a teratocarcinoma at the histological examination, showed an RTE score 2 related to its multilocular cyst-like appearance, with thick septa. Its macrostructure with a prevalence of fluid-filled areas probably does not allow it to be characterised as a malignant tumour at the RTE evaluation.

In the group of 41 pseudonodular lesions no difference was noticed in the RTE statistical data according to size.

Overall, the results of the elastograms gave $28 \mathrm{TP}, 110$ TN, 2 FP and $4 \mathrm{FN}$ cases, accounting for $87.5 \%$ sensitivity, 98.2\% specificity, 93.3\% PPV, 96.4\% NPV and 95.8\% accuracy in differentiating malignant from benign lesions in the 144 nodules/pseudo-nodules (Table 6).

\section{Discussion}

Clinical examination has been the method for assessing patients with acute scrotal pain and for detecting scrotal 
Fig. 2 a Case \# 1: Ultrasound showed a small hypoechoic nodule ( $3 \mathrm{~mm}$ in size), with homogeneous elasticity (score 1) at RTE (arrow). Follow-up highlighted the ability of RTE to assess the benign aspect. b Case \# 2: a 4-mm hypoechoic nodule at ultrasound, completely anaelastic (score 4) at RTE (arrowhead), was considered suspicious. A close follow-up carried out at regular intervals for 30 months showed unchanged size and appearance. The lesion has therefore been reclassified as "probably benign" and its inelastic aspect as a false-positive of RTE
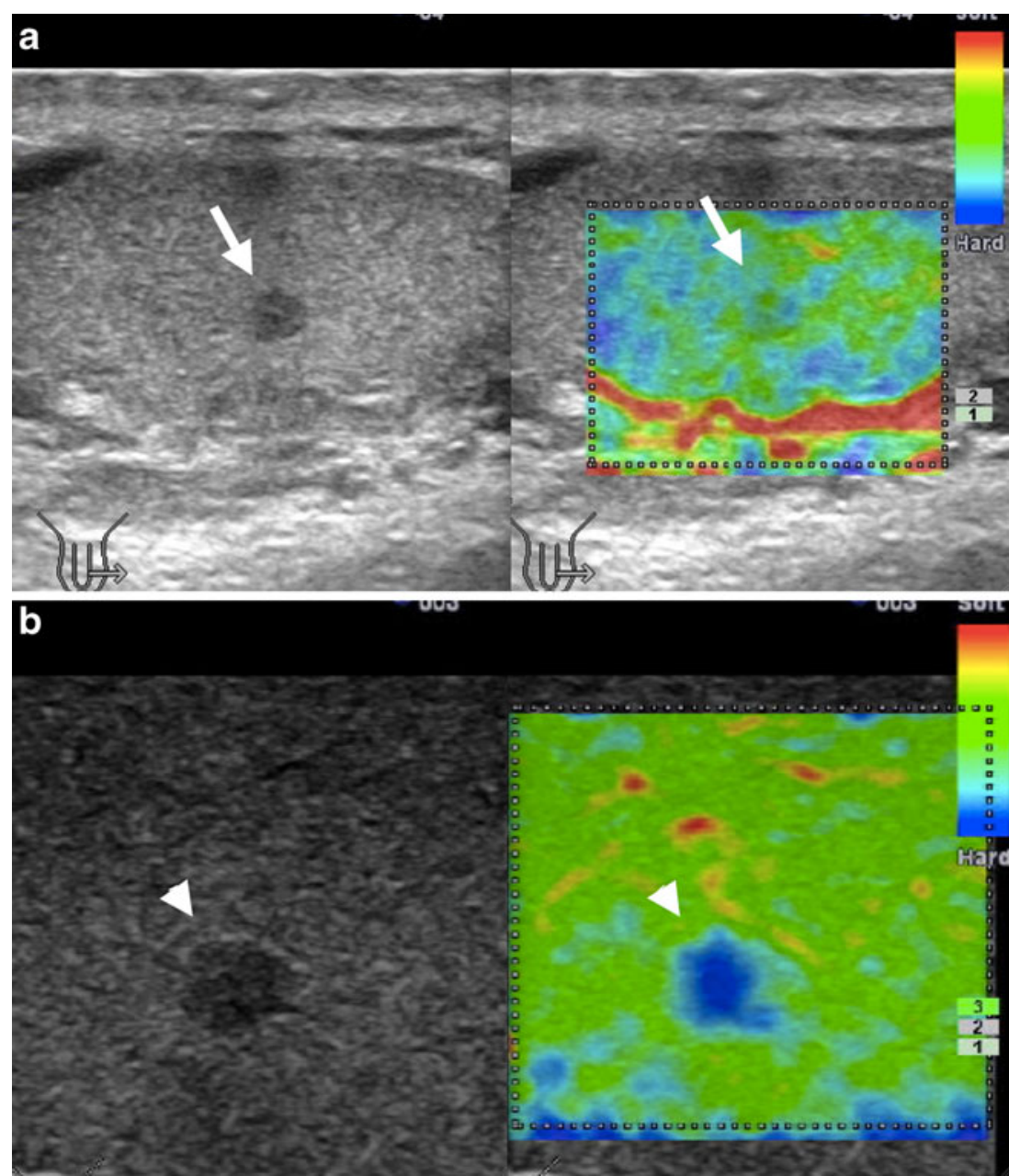

masses. Unfortunately palpation is highly related to the examiner's experience and to the lesion's size. The advent of ultrasound, which allows even small lesions to be identified, has exceeded these limits. Ultrasound is now the imaging technique of choice for evaluating scrotal abnormalities [2]. The primary function of ultrasound in the diagnosis of a testicular mass is to distinguish intratesticular from extratesticular location because most extratesticular masses are benign, but intratesticular ones are malignant [1]. Even though ultrasound is extremely sensitive, it does not offer any further information about the nature of the lesion, especially small ones. The detection of hypoechoic intratesticular lesions $<5 \mathrm{~mm}$ is not infrequent using transducers with a frequency range of $7.5-14 \mathrm{MHz}$ or greater. It raises the issue of how to manage these nodules and how often and for how long these patients should be followed to exclude the tumoural nature of the nodules.

In an attempt to characterise intratesticular lesions, the use of colour Doppler was introduced in the past. The background was the increased microvascularity associated with cancer growth. Unfortunately the presence of abnormally increased flow within testicular nodules depends on their size rather than their cell type and the evidence has shown that only masses larger than $1.5 \mathrm{~cm}$ are likely to have demonstrable flow [17].

Recently, Real-time Elastography was introduced to evaluate relative tissue displacement after slight compression [5]. The total amount of displacement used to compute the strain may be the sum of the external compression plus physiological patient motion; even the minimal displacement of the testis due to the cremaster muscle contraction may contribute to the strain imaging.

The real time evaluation permits a quick visual representation of the strain distribution at a good spatial resolution. The spatial resolution of Sonoelastography is dependent on the ultrasonic frequency, the pulse length and, mainly, on the length of the correlation window. Anyhow, the smallest window that can be adjusted corresponds to one pixel on the monitor; thus actually, the resolution for diagnosis is in the range of $1 \mathrm{~mm}$.

At the RTE evaluation the normal testicles show mainly a medium level of elasticity (displayed in green); some linear "red" structures within the testes are related to fluid component (i.e. small vessels not often visible on the B- 



Fig. 3 a Ultrasound showed a nodule (14 mm in size) slightly hyperechoic $(*)$ compared with the testicular tissue. b Unusual appearance on RTE: the central strain area $(*)$ was surrounded by a nearly complete no strain - stiff - rim (arrowheads; i.e. the peripheral part of the lesion was blue, the central part was green). It was must classified as Score 2. The relative elasticity (i.e. displacement) of different tissue showed by RTE, explains the apparent softer aspect of the anterior portion of the rim (displayed in light blue instead of dark blue) and of the adjacent testicle (displayed in red). This is the

mode image - Fig. 1). Sometimes the glandular tissue below the tunica albuginea of the testicle presents less relative strain, displayed in light blue, probably due to the limited tissue displacement determined by the fibrous covering. The pressure applied with the probe may influence this aspect; nevertheless the amount of the relative stiffness does not usually affect the assessment of the lesion. Specifically, if pressure increases above a certain level, the elastic image starts to change drastically and the operator avoids immediately using higher levels of pressure [6].

RTE has already demonstrated its promising value in the detection and differentiation of nodules in the breast, prostate, thyroid gland and lymph nodes [7-13]. However, there are only a few reports available in the literature regarding the efficacy of real-time tissue elastography for scrotal mass evaluation [14-16, 18].

In 2005 Pallwein et al. [14] reported their preliminary findings of the value of RTE in the differential diagnosis of consequence of the higher tissue displacement generated in the near field by the applied compressional forces. c Colour Doppler showed mainly peripheral vascularisation. d Pathological specimens of the testes: Leydig tumour. Central area of the nodule: polygonal cells with abundant, eosinophilic cytoplasm, solid, sheet-like pattern and no interstitial fibrous stroma. Haematoxylin-Eosin $(\times 100)$. e Peripheral area of the nodule: sclerohyalinosis of the testicular tissue (arrowheads) adjacent to the neoplasm $(*)$; peripheral vessels surrounding the tumor are visibile (arrows; $\times 100)$

inflammatory and neoplastic disease of the testicles in 15 patients. They concluded that RTE improved the detection of testicular masses and allowed the distinction of inflammatory changes from other pathological processes based on the difference in tissue elasticity.

In 2010 Grasso et al. [16] pointed to the inability to differentiate malignant and benign lesions on the basis of elastography only, because the pattern was quite similar. Further systematic experience was advocated to improve the assessment of testicular lesions.

The current study investigated the ability of RTE in assessing testicular lesions, distinguishing benign from malignant. In comparison to a study conducted by Grasso et al. [16] we classified nodules on the basis of a coloured elastographic score according to the distribution and degree of strain, as suggested by Itoh et al. [6].

An elasticity score of 1 indicates that lesions have the same compressibility of the sorrounding tissue. In our study RTE has shown a prevalence of score 1 in $93.7 \%$ of the benign 
Fig. 4 a Ultrasound showed a subcapsular oval-shaped hypoechoic pseudonodule (arrow) and an adjacent rounded hyperechoic spot in a patient with recent scrotal trauma. At RTE the entire lesion and its surrounding area were blue (arrowhead), indicating a score 5 appearance that usually indicates suspected neoplasm. Considering the shape and the recent history of trauma, a close follow-up was planned. b Six months later the B-mode image seemed to be about the same (arrow), but at RTE half of the nodule showed regular strain and the surrounding stiffness had disappeared (arrowhead). c After 9 months it was reduced in size, partially calcified (arrow) and became elastic (score 1; arrowhead)


lesions and only one case with score 1 was found malignant. Although our findings will require further confirmation, this result suggests that lesions with score 1 , lesser than $10 \mathrm{~mm}$, could be considered benign unless biochemical data/tumoral markers/hormonal levels suggest otherwise.
Elasticity scores of 2 indicate lesions that are mainly soft, but have some stiff areas compared with the normal tissue. Itoh et al. [6] assessing breast nodules considered this pattern often characteristic of benign lesions such as fibroadenoma. In Itoh's study only $21 \%$ of score 2 lesions 
Table 4 RTE results - statistical data in differentiating malignant from benign lesions regarding to their shape

\begin{tabular}{lll}
\hline & Nodules & Pseudo-nodules \\
\hline Sensitivity & $87.5 \%$ & $0 \%$ \\
Specificity & $97.1 \%$ & $100 \%$ \\
Positive predictive value & $93.3 \%$ & $0 \%$ \\
Negative predictive value & $94.5 \%$ & $100 \%$ \\
Accuracy & $94.1 \%$ & $100 \%$ \\
\hline
\end{tabular}

were malignant. In our study $37.5 \%$ of score 2 lesions were malignant, two of which had a diameter larger than $11 \mathrm{~mm}$. Therefore we suggest that when assessing scrotal lesions with such pattern (i.e. score 2) they should be considered suspicious until proven otherwise if larger than $11 \mathrm{~mm}$ and should be closely monitored if less than $10 \mathrm{~mm}$ diameter.

An elasticity score of 3 indicates strain at the periphery of a lesion with no strain at the centre. There were no score 3 pattern cases in our study. The absence of score 3 lesions may be explained by two main factors. Firstly, Itoh's original score was invented for the breast and lesions in the breast have a considerably different histological pattern - and therefore RTE pattern - compared with testicular lesions. Secondly, the elasticity score of 3, was mainly found by Itoh in benign lesions of the breast [6]. In particular benign nodules larger than $10 \mathrm{~mm}$ may be found quite often in the breast, but this doesn't happen in the testicle, where the vast majority of large nodules are nearly always malignant. Further studies to confirm these statements will be necessary.

An elasticity score of 4 describes a circumscribed and homogeneously harder nodule than the adjacent tissue and indicates no strain in the entire lesion. This is considered characteristic of malignancy. An elasticity score of 5, which shows no strain in the entire lesion and the surrounding area, indicates infiltration of cancer cells into the interstitial tissues [6]. The analysis of the data relative to the strain characteristic of the malignant lesions in our study shows a prevalence of score 4 and 5 patterns (only one lesion showed score 5 pattern) in $87.5 \%$ of the cases The prevalence of a stiff pattern may be explained by the fact that seminoma - the commonest histotype of testicular

Table 5 RTE results - statistical data in differentiating malignant from benign lesions regarding to their size

\begin{tabular}{llll}
\hline & $\begin{array}{l}\text { Nodules } \\
<5 \mathrm{~mm}\end{array}$ & $\begin{array}{l}\text { Nodules } \\
6-10 \mathrm{~mm}\end{array}$ & $\begin{array}{l}\text { Nodules } \\
>11 \mathrm{~mm}\end{array}$ \\
\hline Sensitivity & $100 \%$ & $88.2 \%$ & $81.8 \%$ \\
Specificity & $98.5 \%$ & $100 \%$ & $100 \%$ \\
Positive predictive value & $80 \%$ & $100 \%$ & $100 \%$ \\
Negative predictive value & $100 \%$ & $66.6 \%$ & $0 \%$ \\
Accuracy & $98.8 \%$ & $90.4 \%$ & $81.8 \%$ \\
\hline
\end{tabular}

neoplasm - usually presents high cellularity of typical seminoma cells associated with discrete interstitial stroma and even a thick fibrous pseudocapsule (Fig. 5d).

Regarding Leydig tumours there are some important aspects to be considered. Both cases in our study were slightly hyperechoic compared with the surrounding tissue and showed mainly an elastic pattern at RTE. At histology Leydig tumour consists of polygonal cells with abundant, eosinophilic cytoplasm, solid, sheet-like pattern without interstitial fibrous stroma. The progressive growth of this type of tumour induces sclerohyalinosis of the surrounding glandular tissue detectable as a stiff blue rim at RTE (Fig. 3d). On the basis of the correlation between histology and RTE pattern there could be a possibility of suspecting different malignant histotypes with non-invasive investigation. This could be useful in planning more conservative surgery in selected cases. Therefore we suggest introducing, as part of the assessment of testicular lesions, a new subscore that could be related to a Leydig tumour. Considering that the RTE appearance described seems the exact opposite of the known score 3 reference, we suggest calling it "score 3 inverted". Undoubtedly further studies will be needed to confirm the correspondence of this pattern with the Leydig tumour, especially in small nodules.

The analysis of the data relative to the nodule size has shown the particular usefulness of RTE in the assessment of small testicular nodules. Despite the statistical data results account the highest specificity and positive predictive value in nodules larger than $11 \mathrm{~mm}$, the role of RTE in these nodules seems less useful because most of them were malignant at clinical and ultrasound assessment.

All the pseudo-nodular areas were elastic and well characterisable with RTE. We did not observe any variation during the follow-up. This result suggests that follow up may be not necessary for patients who have pseudo-nodular lesions with score 1 .

Despite advantages and significative potentiality RTE has limitations that need to be considered. The main limitation of RTE is related to the manual compression source by which the tissue is displaced to gain the strain. As the driving force is not exactly known, RTE may only assess the compressibility ratio of different tissues and consequently the average relative elasticity, not their absolute value. The strain value is a relative index that may change with tissue composition, tissue structure and the different degree of compression. The latter is greatly operator-dependent and may affect intra and interobserver variability. RTE provides only qualitative information whose interpretation may be influenced by the experience of the operator. Even the use of a strain ratio analysis, allows only a semiquantitive evaluation. Pure quantification systems of the tissue elasticity might be helpful in the future to determine clearly measurable thresholds. 
Fig. 5 a Multiple hypoechoic testicular nodules at ultrasound (*). b Colour Doppler of the largest nodule showed mainly peripheral vascular signals. c Longitudinal and transverse testicular imaging. The nodules $(*)$, score $4-5$ at RTE, were correctly identified as tumours. d Pathological specimen. Multifocal seminoma of the testis at histological diagnosis: high cellularity, typical seminoma cells $\left(^{*}\right)$ with discrete interstitial stroma (arrowheads) and thick fibrous pseudocapsule (arrow; $\times 40$ )
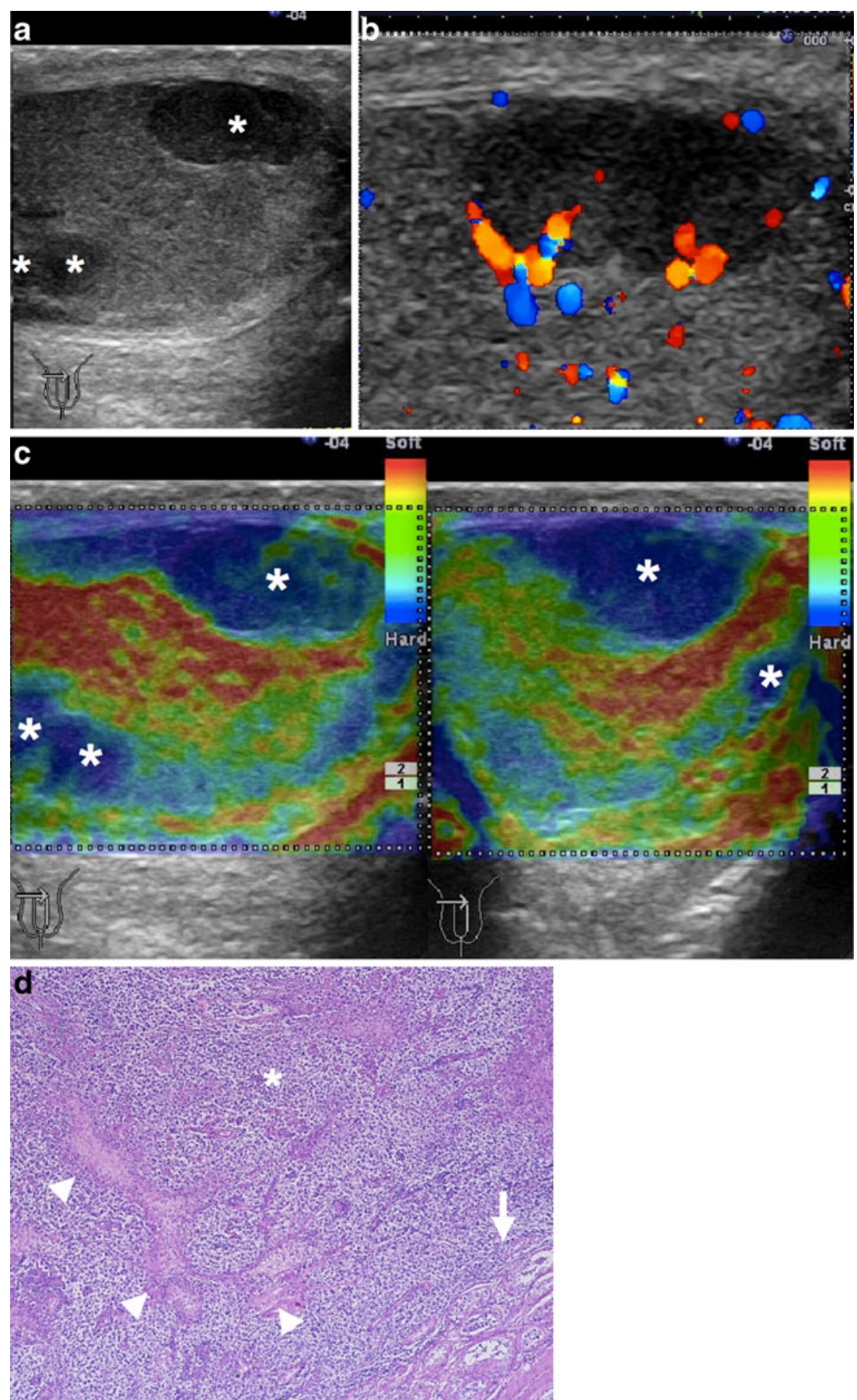

In conclusion, our study has shown RTE to have a good sensitivity and specificity in differentiating malignant from benign lesions. RTE was a very useful technique in assessing small testicular nodules and all types of pseusonodules and could be helpful in deciding the most appropriate clinical approach, allowing in particular expectant management in selected cases. The role of RTE is less relevant for larger nodules because most of them are malignant at clinical and ultrasound assessment, limiting RTE to a simply confirmatory role.
Table 6 Overall RTE results - statistical data in differentiating malignant from benign lesions in 144 nodules/pseudo-nodules

\section{Sensitivity}

$87.5 \%$

Specificity

$98.2 \%$

Positive predictive value

$93.3 \%$

Negative predictive value

$96.4 \%$

Accuracy 
Lastly, RTE is time-efficient and may be easily performed during a conventional ultrasound examination.

Open Access This article is distributed under the terms of the Creative Commons Attribution Noncommercial License which permits any noncommercial use, distribution, and reproduction in any medium, provided the original author(s) and source are credited.

\section{References}

1. Ragheb D, Higgins JL Jr (2002) Ultrasonography of the scrotum: technique, anatomy and pathologic entities. J Ultrasound Med 21:171-185

2. Mihmanli I, Kantarci F (2009) Sonography of scrotal abnormalities in adults: an update. Diagn Interv Radiol 15:64-73

3. Krouskop TA, Dougherty DR, Vinson FS (1987) A pulsed Doppler ultrasonic system for making noninvasive measurements of the mechanical properties of soft tissue. J Rehabil Res Dev 24:1-8

4. Ophir J, Céspedes I, Ponnekanti H, Yazdi Y, Li X (1991) Elastography: a quantitative method for imaging the elasticity of biological tissues. Ultrason Imaging 13:111-134

5. Shiina T, Nitta N, Ueno E, Bamber JC (2002) Real time tissue elasticity imaging using the combined autocorrelation method. J Med Ultrasonics 29:119-128

6. Itoh A, Ueno E, Tohno E, Kamma H, Takahashi H, Shiina T, Yamakawa M, Matsumura T (2006) Breast disease: clinical application of US elastography for diagnosis. Radiology 239:341-350

7. Pallwein L, Mitterberger MJ, Struve P, Horninger W, Aigner F, Bartsch G, Gradl J, Schurich M, Pedross F, Frauscher F (2007) Comparison of sonoelastography guided biopsy with systematic biopsy: impact on prostate cancer detection. Eur Radiol 17:2278-2285

8. Miyagawa T, Tutsumi M, Matsumura T, Shimokama T, Miyanagas N, Akazas H (2009) Real-time elastography for the diagnosis of the prostate cancer: evaluation of elastographic moving images. Jpn J Clin Oncol 39:394-398

9. Konig K, Scheipers U, Pesavento A, Lorenz A, Ermert H, Senge $\mathrm{T}$ (2005) Initial experiences with real-time elastography guided biopsies of the prostate. J Urol 174:115-117

10. Rizzatto G, Aiani L, Baldassarre S, Bulzacchi A, Della Sala S, Locatelli $\mathrm{M}$ et al (2006) Characterization of breast lesions with real-time sonoelastography: results from the Italian Multicenter Clinical Trial. Abstract -RSNA 2006. Chicago. USA

11. Zhi H, Ou B, Luo BM, Feng X, Wen YL, Yang HY (2007) Comparison of ultrasound elastography, mammography, and sonography in the diagnosis of solid breast lesions. J Ultrasound Med 26:807-815

12. Lyshchik A, Higashi T, Asato R, Tanaka S, Ito J, Mai JJ, PellotBarakat C, Insana MF, Brill AB, Saga T, Hiraoka M, Togashi K (2007) Thyroid gland tumor diagnosis at US elastography. Radiology 237:202-211

13. Lyshchik A, Higashi T, Asato R, Tanaka S, Ito J, Hiraoka M, Insana MF, Brill AB, Saga T, Togashi K (2007) Cervical lymph node metastases: diagnosis at sonoelastography - initial experience. Radiology 243:258-267

14. Pallwein L, Pallwein E, Schurich M, Fischbach V, Steiner H, Frauscher F (2005) Sonoelastography of the testicles: preliminary results in the diagnosis of different pathological processes. Abstract -Fourth International Conference on the Ultrasonic Measurement and Imaging of Tissue Elasticity. Austin - Texas, USA

15. Shah A, Rajayogeswaran B, Sellars ME, Sidhu PS (2009) Imaging the testis using real-time tissue elastography - seeing what you feel? A pictorial review. Abstract - Proceedings of the 21st Euroson Congress. Edinburgh

16. Grasso M, Blanco S, Raber M, Nespoli L (2010) Elasto-sonography of the testis: preliminary experience. Arch Ital Urol Androl 82:160163

17. Oyen RH (2002) Scrotal ultrasound. Eur Radiol 12:19-34

18. Schurich M, Aigner F, Frauscher F, Pallwein L (2009) The role of ultrasound in assessment of male fertility. Eur J Obstet Gynecol Reprod Biol 144(Suppl 1):S192-S198 